\title{
Role of elevated liver transaminase enzymes in diagnosis of liver injury in cases after abdominal trauma
}

\author{
Adel H. Elbaih ${ }^{1}$, Mohamed A. Ali ${ }^{1}$, Ahmed Abozaid ${ }^{1}$, Bassem M. Saady ${ }^{1}$, Sherif H. Farrag ${ }^{2}$
}

${ }^{1}$ Department of Emergency Medicine, Suez Canal University, Ismailia, Egypt

${ }^{2}$ Department of General Surgery, Suez Canal University, Ismailia, Egypt

Received: 06 April 2016

Accepted: 10 May 2016

\author{
*Correspondence: \\ Dr. Adel H. Elbaih, \\ E-mail: elbaihzico@yahoo.com
}

Copyright: () the author(s), publisher and licensee Medip Academy. This is an open-access article distributed under the terms of the Creative Commons Attribution Non-Commercial License, which permits unrestricted non-commercial use, distribution, and reproduction in any medium, provided the original work is properly cited.

\begin{abstract}
Background: Major liver trauma in polytraumatic patients accounts for significant morbidity and mortality. Delayed diagnosis of patients with severe liver injuries is associated with an adverse outcome. Diagnostic modalities include serial abdominal examination, focused abdominal sonography for trauma, computed tomography scanning, diagnostic peritoneal lavage. The objective of this study was to detect relationship between raise of serum levels of hepatic transaminase enzymes and liver injury, and if the degree of the rise may also correlate with the severity of hepatic injury after abdominal trauma.

Methods: cross sectional, prospective study observational study of all patients admitted emergency department in Suez Canal university hospital. Patients with abdominal trauma were subdivided (according to either surgical record after abdominal exploration or CT scans reports released by radiology department) in two groups, group with liver injury (31) cases and those without liver injuries (31) cases.

Results: As regard to alanine aminotransferases (ALT) our study showed that serum ALT was raised in 28 cases from 31 cases of liver injury and only 4 patients with raised serum ALT did not have liver trauma (sensitivity: 90.3\%, specificity: $87 \%$, CI: $88.7 \%$ ). Regarding aspartate aminotransferases (AST) our study showed serum AST was raised in about 21 cases from 31 cases of liver injury and only 7 patients with raised serum AST did not have liver trauma (sensitivity: $93.5 \%$, specificity: $77.4 \%$, CI: $87.1 \%$ ).

Conclusions: An abnormal ALT and AST can predict the presence of hepatic injuries in abdominal trauma patients, while the level of the abnormality can determine the severity of the insult to the liver. Also we can conclude that ALT is more specific for liver injury more than AST, Although AST show high sensitivity for liver injury than ALT.
\end{abstract}

Keywords: Liver transaminase enzymes, Liver injury, Abdominal trauma

\section{INTRODUCTION}

Major liver trauma in polytraumatic patients accounts for significant morbidity and mortality. Delayed diagnosis of patients with severe liver injuries is associated with an adverse outcome. Diagnostic modalities include serial abdominal examination, focused abdominal sonography for trauma, computed tomography scanning, diagnostic peritoneal lavage. ${ }^{1}$
Trauma represents the third leading cause of death worldwide and the leading cause of death among children, and young adults aged 1 to 44 years. ${ }^{2}$ Despite the relative protection of liver by the rib cage, its large size and fragile tissue, make the liver the second most commonly injured organ following abdominal trauma, and constitute $5 \%$ of all cases hospitalized for trauma.

Early diagnosis of the nature and extent of liver injury may result in significant decrease in number of morbidity and mortality. ${ }^{5}$ Diagnostic modalities include serial 
abdominal examination, FAST, C.T scan, D.P.L. ${ }^{6}$ Therapeutic options for hepatic trauma include both nonoperative and operative management.

Liver transaminases enzymes (AST and ALT) are present in high concentrations in hepatocytes, and can be used as specific indicators of hepatocellular necrosis. They may be released into the circulation in large quantities following acute liver injury or inflammation. ${ }^{7}$

\section{METHODS}

The design of this study was all abdominal trauma patients attended Emergency department in Suez Canal University hospital in cross sectional, prospective study.

These patients with abdominal trauma will be subdivided (according to either surgical record after abdominal exploration or CT scans reports released by radiology department) in two groups, group with liver injury (31) cases and those without liver injuries (31) cases. Abdominal trauma may be penetrating or blunt trauma, also may be isolated or polytrauma.

Laboratory tests aspartate aminotransferase (AST), alanine minotransferase (ALT), will be done as aroutine investigation on admission at emergency room as initial set, then will be repeated after 6 hours, 24 hours from time of injury and third, fifth days from admission, results will be collected. The values will compared with reference ranges for our hospital

A standardized data collection form (sheet) is made for each trauma patient during the hospital admission included, name, sex, time, date, place of injury, mechanism of injury, vital signs including (heart rate, blood pressure, and respiratory rate), clinical examination, injury severity score (ISS), investigation, grade of liver injury, length of stay in ICU, total inpatient length of stay, and outcome data.

\section{Data analysis}

Data was collected throughout history, clinical examination and radiological investigations were coded, entered and analyzed using Microsoft Excel software. Gathered data was then imported into SPSS (Statistical Package for Social Sciences) software program version 13.0 for analysis.
Data will be presented in the form of graphs, numeric presentations and tubular presentations.

\section{RESULTS}

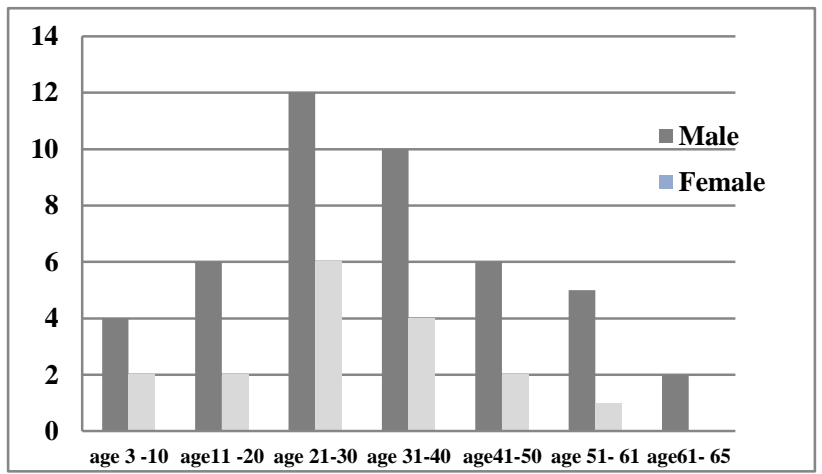

Figure 1: Distribution according to age.

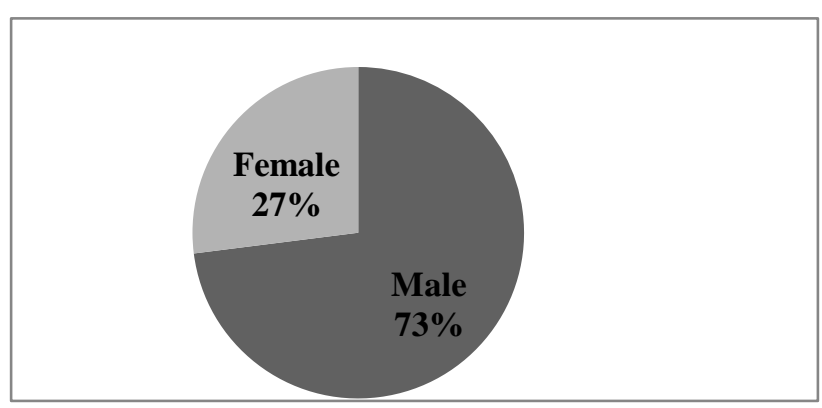

Figure 2: Pie chart of distribution according to gender.

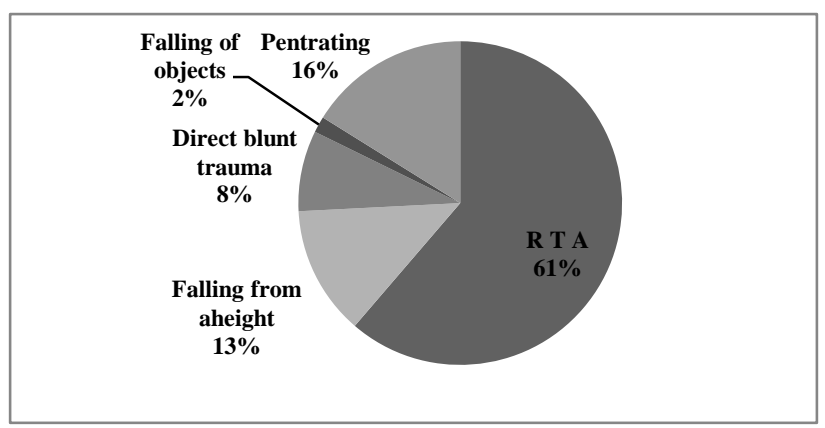

Figure 3: Pie charts of distribution according to type of trauma.

Table 1: Comparison between the studied groups as regard vital signs.

\begin{tabular}{|lllll|}
\hline Demographic data & Liver injury & Non liver injury & Test & p-value (sig.) \\
\hline Heart rate $(\mathbf{b} / \mathbf{m})$ & $\mathbf{( N = 3 1 )}$ & $\mathbf{( N = 3 1 )}$ & \multirow{2}{*}{$1.014^{*}$} & 0.315 (NS) \\
\hline Mean \pm SD & $106.38 \pm 21.40$ & $101.06 \pm 19.88$ & & \\
\hline Median (range) & $105(68-150)$ & $100(70-140)$ & & \\
\hline Systolic blood pressure (mmHg) & & & & \\
\hline
\end{tabular}




\begin{tabular}{|c|c|c|c|c|}
\hline Mean \pm SD & $101.29 \pm 19.57$ & $102.74 \pm 19.52$ & \multirow{2}{*}{$-0.292^{*}$} & \multirow{2}{*}{$0.771(\mathrm{NS})$} \\
\hline Median (range) & $100(60-130)$ & $100(60-140)$ & & \\
\hline \multicolumn{5}{|c|}{ Diastolic blood pressure (mmHg) } \\
\hline Mean \pm SD & $62.45 \pm 14.41$ & $65.32 \pm 9.56$ & \multirow{2}{*}{$-0.734 \bullet$} & \multirow{2}{*}{$0.463(\mathrm{NS})$} \\
\hline Median (range) & $60(30-80)$ & $70(40-80)$ & & \\
\hline \multicolumn{5}{|c|}{ Respiratory rate (min) } \\
\hline Mean \pm SD & $16.06 \pm 4.27$ & $14.70 \pm 3.13$ & \multirow{2}{*}{$-1.005 \bullet$} & \multirow{2}{*}{0.315 (NS) } \\
\hline Median (range) & $15(11-25)$ & $14(10-23)$ & & \\
\hline \multicolumn{5}{|c|}{ Body temperature $\left({ }^{\circ} \mathrm{C}\right)$} \\
\hline Mean \pm SD & $37.08 \pm 0.27$ & $37.33 \pm 0.28$ & \multirow{2}{*}{$-3.339 \bullet$} & \multirow{2}{*}{0.001 (HS) } \\
\hline Median (range) & $37(36.50-37.80)$ & $37.30(36.90-38)$ & & \\
\hline
\end{tabular}

$\mathrm{p}<0.05$ is significant; Sig.: significance.

Table 2: Comparison between the studied groups as regard general signs.

\begin{tabular}{|c|c|c|c|c|c|c|}
\hline \multirow{3}{*}{ General signs } & \multirow{2}{*}{\multicolumn{2}{|c|}{$\begin{array}{l}\text { Liver injury } \\
(\mathbf{N}=\mathbf{3 1})\end{array}$}} & \multirow{2}{*}{\multicolumn{2}{|c|}{$\begin{array}{l}\text { Non liver injury } \\
(\mathbf{N}=\mathbf{3 1})\end{array}$}} & \multirow{3}{*}{ Test: } & \multirow{3}{*}{ p-value (Sig.) } \\
\hline & & & & & & \\
\hline & No. & $\%$ & No. & $\%$ & & \\
\hline Pallor & 18 & $58.1 \%$ & 16 & $51.6 \%$ & 0.261 & $0.610(\mathrm{NS})$ \\
\hline Irritability & 11 & $35.5 \%$ & 12 & $38.7 \%$ & 0.069 & 0.793 (NS) \\
\hline \multicolumn{7}{|l|}{ Shock index } \\
\hline A & 12 & $38.7 \%$ & 16 & $51.6 \%$ & \multirow{3}{*}{1.611} & \multirow{3}{*}{0.447 (NS) } \\
\hline B & 13 & $41.9 \%$ & 12 & $38.7 \%$ & & \\
\hline $\mathrm{C}$ & 6 & $19.4 \%$ & 3 & $9.7 \%$ & & \\
\hline
\end{tabular}

$\mathrm{p}<0.05$ is significant; Sig.: significance.

The study was carried out on 62 patients. There ages were from 3 to 65 years with mean of study 30 years, and $45(72.6 \%)$ of them were males and $17(27.0 \%)$ were females; the peak incidence $(29 \%)$ was in the third decades of life followed by fourth decads of life (22\%).

Table 3: Imaging findings in liver injury group.

\begin{tabular}{|c|c|c|}
\hline \multirow[t]{2}{*}{ Imaging findings } & \multicolumn{2}{|c|}{$\begin{array}{l}\text { Liver injury } \\
\qquad(\mathbf{N}=\mathbf{3 1})\end{array}$} \\
\hline & No. & $\%$ \\
\hline \multicolumn{3}{|c|}{ Grade of liver injury } \\
\hline Minor liver injury & (13) & $(41.9 \%)$ \\
\hline Grade I & 5 & $16.1 \%$ \\
\hline Grade II & 8 & $25.8 \%$ \\
\hline Major liver injury & (18) & $(58.1 \%)$ \\
\hline Grade III & 9 & $29 \%$ \\
\hline Grade IV & 6 & $19.4 \%$ \\
\hline Grade V & 3 & $9.7 \%$ \\
\hline \multicolumn{3}{|c|}{ Type of liver injury } \\
\hline Hematoma & 16 & $51.6 \%$ \\
\hline Laceration & 15 & $48.4 \%$ \\
\hline \multicolumn{3}{|l|}{ Site of injury } \\
\hline Subcapsular & 18 & $58.1 \%$ \\
\hline Parenchymal & 13 & $41.9 \%$ \\
\hline
\end{tabular}

The most cause of abdominal trauma is blunt abdominal trauma 52 patients $(84 \%)$, and the most cause of blunt abdominal trauma was road traffic accident in 38 patients $(61.3 \%)$ in form of motor car accidents $(37 \%)$, motor cycle crush $(19.4 \%)$, and bicycle accidents $(4.8 \%)$.
Followed 8 patients $(12.9 \%)$ suffered from falling from height and 5 patients $(8.1 \%)$ suffered from direct blunt trauma and one patient $(1.6 \%)$ suffered from falling of heavy objects. Penetrating trauma patients 10 patients accounts about $16.1 \%$ of all trauma patients.

Table 4: Incidence of organ injuries in non-liver injury group.

\begin{tabular}{|lll|}
\hline \multirow{2}{*}{ Organ injuries } & \multicolumn{2}{c|}{ Non liver injury } \\
\cline { 2 - 3 } & \multicolumn{2}{|c|}{$\mathbf{( N = 3 1 )}$} \\
\hline $\begin{array}{l}\text { Sign of abdominal trauma without } \\
\text { organs injury }\end{array}$ & $(5)$ & $(16.1 \%)$ \\
\hline Injury of one organ & $(22)$ & $(70.9 \%)$ \\
\hline Spleen injuries & 12 & $38.7 \%$ \\
\hline Renal injuries & 3 & $9.7 \%$ \\
\hline Mesentric tear & 2 & $6.5 \%$ \\
\hline Retroperitoneal hematoma & 2 & $6.5 \%$ \\
\hline Small bowel injuries & 2 & $6.5 \%$ \\
\hline Pancreatic injuries & 1 & $3.2 \%$ \\
\hline Injury of more than one organ & $(4)$ & $(13 \%)$ \\
\hline Spleen + upper pole of left kidney & 1 & $3.2 \%$ \\
\hline Spleen + tear small intestine & 1 & $3.2 \%$ \\
\hline $\begin{array}{l}\text { Bladder injury + retroperitoneal } \\
\text { hematoma }\end{array}$ & 1 & $3.2 \%$ \\
\hline $\begin{array}{l}\text { Colon tear +mesenteric injury+ Rt } \\
\text { kidney }\end{array}$ & 1 & $3.2 \%$ \\
\hline
\end{tabular}


Table 5: Comparison between the studied groups as regard ALT.

\begin{tabular}{|c|c|c|c|c|}
\hline ALT & $\begin{array}{l}\text { Liver injury } \\
(\mathbf{N}=\mathbf{3 1})\end{array}$ & $\begin{array}{l}\text { Non liver injury } \\
(\mathbf{N}=\mathbf{3 1})\end{array}$ & Test• & p-value (Sig.) \\
\hline \multicolumn{5}{|l|}{ Initial } \\
\hline Mean \pm SD & $66.38 \pm 33.87$ & $32.22 \pm 8.44$ & \multirow{2}{*}{-4.161} & \multirow{2}{*}{$<0.001$ (HS) } \\
\hline Median(range) & $66(23-164)$ & $33(17-48)$ & & \\
\hline \multicolumn{5}{|l|}{6 hour } \\
\hline Mean \pm SD & $128.29 \pm 106.01$ & $41 \pm 14.44$ & \multirow{2}{*}{-5.584} & \multirow{2}{*}{$<0.001$ (HS) } \\
\hline Median (range) & $90(30-480)$ & $39(18-89)$ & & \\
\hline \multicolumn{5}{|l|}{24 hour } \\
\hline Mean \pm SD & $292.93 \pm 343.27$ & $43.29 \pm 21.17$ & \multirow{2}{*}{-5.584} & \multirow{2}{*}{$<0.001$ (HS) } \\
\hline Median (range) & $120(35-1404)$ & $38(17-102)$ & & \\
\hline \multicolumn{5}{|l|}{$3^{\text {rd }}$ day } \\
\hline Mean \pm SD & $447.03 \pm 772.24$ & $46.87 \pm 20.20$ & \multirow{2}{*}{-5.738} & \multirow{2}{*}{$<0.001(\mathrm{HS})$} \\
\hline Median (range) & $190(33-4270)$ & $40(22-106)$ & & \\
\hline \multicolumn{5}{|l|}{$5^{\text {th }}$ day } \\
\hline Mean \pm SD & $256.61 \pm 268.09$ & $40.80 \pm 11.59$ & \multirow{2}{*}{-5.768} & \multirow{2}{*}{$<0.001$ (HS) } \\
\hline Median (range) & $160(32-1200)$ & $39(22-76)$ & & \\
\hline
\end{tabular}

$\mathrm{p}<0.05$ is significant; Sig.: significance.

Table 6: Comparison between the studied groups as regard AST.

\begin{tabular}{|c|c|c|c|c|}
\hline AST & $\begin{array}{l}\text { Liver injury } \\
(\mathbf{N}=\mathbf{3 1})\end{array}$ & $\begin{array}{l}\text { Non liver injury } \\
(\mathbf{N}=\mathbf{3 1})\end{array}$ & Test• & p-value (Sig.) \\
\hline \multicolumn{5}{|l|}{ Initial } \\
\hline Mean \pm SD & $80.74 \pm 43.94$ & $35.32 \pm 11.38$ & \multirow{2}{*}{-5.044} & \multirow{2}{*}{$<0.001$ (HS) } \\
\hline Median (range) & $81(22-199)$ & $36(12-63)$ & & \\
\hline \multicolumn{5}{|l|}{6 hour } \\
\hline Mean \pm SD & $146.70 \pm 114.90$ & $45.19 \pm 17.85$ & \multirow{2}{*}{-5.184} & \multirow{2}{*}{$<0.001$ (HS) } \\
\hline Median (range) & $115(27-564)$ & $40(22-79)$ & & \\
\hline \multicolumn{5}{|l|}{24 hour } \\
\hline Mean \pm SD & $306.0 \pm 292.20$ & $53 \pm 32.15$ & \multirow{2}{*}{-5.563} & \multirow{2}{*}{$<0.001$ (HS) } \\
\hline Median (range) & $230(35-1140)$ & $40(20-150)$ & & \\
\hline \multicolumn{5}{|l|}{$3^{\text {rd }}$ day } \\
\hline Mean \pm SD & $453.80 \pm 577.20$ & $51.8 \pm 32.95$ & \multirow{2}{*}{-6.140} & \multirow{2}{*}{$<0.001(\mathrm{HS})$} \\
\hline Median (range) & $290(39-3157)$ & $42(23-199)$ & & \\
\hline \multicolumn{5}{|l|}{$5^{\text {th }}$ day } \\
\hline Mean \pm SD & $287.48 \pm 273.06$ & $43.25 \pm 16.81$ & \multirow{2}{*}{-6.212} & \multirow{2}{*}{$<0.001(\mathrm{HS})$} \\
\hline Median (range) & $190(42-1163)$ & $37(21-105)$ & & \\
\hline
\end{tabular}

$\mathrm{p}<0.05$ is significant; Sig.: significance.

Table 7: Comparison between the studied groups as regard of laboratory investigation.

\begin{tabular}{|c|c|c|c|c|c|c|}
\hline \multirow{3}{*}{ Liver function tests } & \multirow{2}{*}{\multicolumn{2}{|c|}{$\begin{array}{l}\text { Liver injury } \\
(\mathbf{N}=\mathbf{3 1})\end{array}$}} & \multicolumn{2}{|c|}{ Non liver injury } & \multirow{3}{*}{ Test: } & \multirow{3}{*}{ p-value (Sig.) } \\
\hline & & & $(\mathbf{N}=$ & & & \\
\hline & No. & $\%$ & No. & $\%$ & & \\
\hline \multicolumn{7}{|l|}{ ALT } \\
\hline Normal & 3 & $9.7 \%$ & 27 & $87.1 \%$ & \multirow{2}{*}{37.200} & \multirow{2}{*}{$<0.001$ (HS) } \\
\hline Abnormal & 28 & $90.3 \%$ & 4 & $12.9 \%$ & & \\
\hline \multicolumn{7}{|l|}{ AST } \\
\hline Normal & 2 & $6.4 \%$ & 24 & $77.4 \%$ & \multirow{2}{*}{35.457} & \multirow{2}{*}{$<0.001$ (HS) } \\
\hline Abnormal & 29 & $93.5 \%$ & 7 & $22.6 \%$ & & \\
\hline \multicolumn{7}{|l|}{ Bilirubin } \\
\hline Normal & 23 & $74.2 \%$ & 28 & $90.3 \%$ & \multirow{2}{*}{2.763} & \multirow{2}{*}{$0.096(\mathrm{NS})$} \\
\hline Abnormal & 8 & $25.8 \%$ & 3 & $9.7 \%$ & & \\
\hline \multicolumn{7}{|l|}{ ALP } \\
\hline Normal & 18 & $58.1 \%$ & 26 & $83.9 \%$ & \multirow{2}{*}{5.010} & \multirow{2}{*}{$0.025(\mathrm{~S})$} \\
\hline Abnormal & 13 & $41.9 \%$ & 5 & $16.1 \%$ & & \\
\hline \multicolumn{7}{|l|}{ Albumin } \\
\hline Normal & 25 & $80.6 \%$ & 27 & $87.1 \%$ & 0.477 & $0.490(\mathrm{NS})$ \\
\hline
\end{tabular}




\begin{tabular}{|c|c|c|c|c|c|c|}
\hline Abnormal & 6 & $19.4 \%$ & 4 & $12.9 \%$ & & \\
\hline LDH & & & & & & \\
\hline $\begin{array}{l}\text { Normal } \\
\text { Abnomal }\end{array}$ & $\begin{array}{l}6 \\
25\end{array}$ & $\begin{array}{l}19.4 \% \\
80.06 \%\end{array}$ & $\begin{array}{l}9 \\
22\end{array}$ & $\begin{array}{l}29 \% \\
71 \%\end{array}$ & 0.791 & $0.374(\mathrm{NS})$ \\
\hline Haemoglo & & & & & & \\
\hline $\begin{array}{l}\text { Normal } \\
\text { Abnormal }\end{array}$ & $\begin{array}{l}6 \\
25\end{array}$ & $\begin{array}{l}19.4 \% \\
80.6 \%\end{array}$ & $\begin{array}{l}9 \\
22\end{array}$ & $\begin{array}{l}29 \% \\
71 \%\end{array}$ & 0.791 & $0.374(\mathrm{NS})$ \\
\hline
\end{tabular}

$\mathrm{p}<0.05$ is significant; Sig.: significance.

Table 8: Diagnostic performance of liver function tests for diagnosis of liver injury.

\begin{tabular}{|llllll|}
\hline & SN (95\%CI) & SP (95\%CI) & PPV (95\% CI) & NPV (95\%CI) & ACC (95\% CI) \\
\hline Abnormal ALT & $90.3 \%(79.9-100)$ & $87.1 \%(75.3-98.9)$ & $87.5 \%(76-99)$ & $90 \%(79.3-100)$ & $88.7 \%(80.8-96.6)$ \\
\hline Abnormal AST & $93.8 \%(90.6-100)$ & $77.4 \%(62.7-92.1)$ & $81.1 \%(68.5-93.7)$ & $93 \%(88.3-100)$ & $87.1 \%(78.8-95.4)$ \\
\hline $\begin{array}{l}\text { Abnormal } \\
\text { bilirubin }\end{array}$ & $25.8 \%(10.4-41.2)$ & $90.3 \%(79.9-100)$ & $72.7 \%(46.4-99)$ & $54.9 \%(41.2-68.6)$ & $58.1 \%(45.8-70.3)$ \\
\hline Abnormal ALP & $41.9 \%(24.6-59.3)$ & $83.9 \%(70.9-96.8)$ & $72.2 \%(51.5-92.9)$ & $59.1 \%(44.6-73.6)$ & $62.9 \%(50.9-74.9)$ \\
\hline $\begin{array}{l}\text { Abnormal } \\
\text { albumin }\end{array}$ & $19.4 \%(5.4-33.3)$ & $87.1 \%(75.3-98.9)$ & $60 \%(29.6-90.4)$ & $51.9 \%(38.3-65.5)$ & $53.2 \%(40.8-65.6)$ \\
\hline Abnormal LDH & $80.6 \%(66.7-94.6)$ & $29 \%(13.1-45)$ & $53.2 \%(38.9-67.5)$ & $60 \%(35.2-84.8)$ & $54.8 \%(42.5-67.2)$ \\
\hline
\end{tabular}

Table 9: Comparison between the studied groups as regard of management.

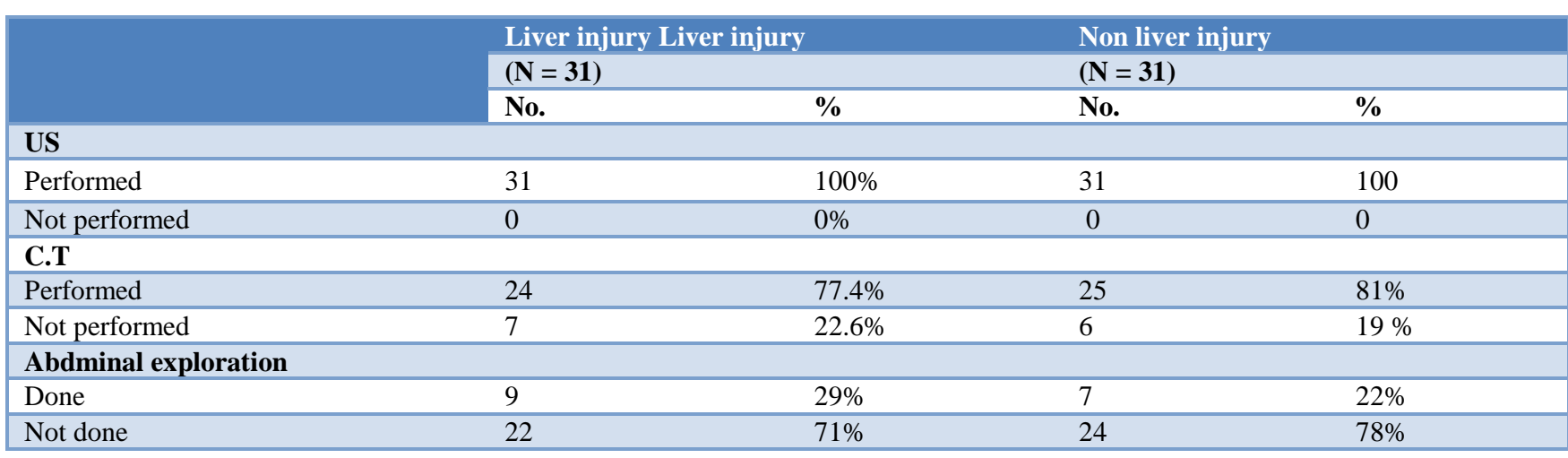

Table 10: Comparison between the studied groups as regard outcome.

\begin{tabular}{|c|c|c|c|c|c|c|}
\hline \multirow{3}{*}{ Outcome } & \multirow{2}{*}{$\begin{array}{l}\text { Liver injury } \\
(\mathbf{N}=\mathbf{3 1})\end{array}$} & \multicolumn{3}{|c|}{ Non liver injury } & \multirow{3}{*}{ Test } & \multirow{3}{*}{ p-value (Sig.) } \\
\hline & & & $(\mathbf{N}=$ & & & \\
\hline & No. & $\%$ & No. & $\%$ & & \\
\hline \multicolumn{7}{|l|}{ ISS } \\
\hline Mean \pm SD & $16.12 \pm 8.81$ & & $14.51 \pm$ & & \multirow{2}{*}{$-0.334 \bullet$} & \multirow{2}{*}{0.739 (NS) } \\
\hline Median (range) & $15(5-35)$ & & 15( & & & \\
\hline \multicolumn{7}{|l|}{ ICU LOS } \\
\hline Mean \pm SD & $2.93 \pm 2.15$ & & 2.38 & & \multirow{2}{*}{$-1.271 \bullet$} & \multirow{2}{*}{0.204 (NS) } \\
\hline Median (range) & $3(0-7)$ & & $2(0$ & & & \\
\hline \multicolumn{7}{|l|}{ Hospital LOS } \\
\hline Mean \pm SD & $7.61 \pm 2.67$ & & $7.9=$ & & \multirow{2}{*}{$-0.702 \bullet$} & \multirow{2}{*}{0.483 (NS) } \\
\hline Median (range) & $7(5-17)$ & & 7.5( & & & \\
\hline \multicolumn{7}{|l|}{ Mortality } \\
\hline Alive & 29 & $93.5 \%$ & 30 & $96.8 \%$ & \multirow{2}{*}{$0.350 \ddagger$} & \multirow{2}{*}{$0.554(\mathrm{NS})$} \\
\hline Died & 2 & $6.5 \%$ & 1 & $3.2 \%$ & & \\
\hline
\end{tabular}


Table 11: Relation between severity of liver injury and ALT.

\begin{tabular}{|c|c|c|c|c|}
\hline ALT & $\begin{array}{l}\text { Minor liver injury } \\
(\mathrm{N}=13)\end{array}$ & $\begin{array}{l}\text { Major liver injury } \\
(\mathrm{N}=18)\end{array}$ & Test• & p-value (Sig.) \\
\hline \multicolumn{5}{|l|}{ Initial } \\
\hline Mean \pm SD & $56.61 \pm 36.25$ & $88.44 \pm 31.17$ & \multirow{2}{*}{-1.825} & \multirow{2}{*}{$0.068(\mathrm{NS})$} \\
\hline Median (range) & $45(29-164)$ & $87(23-138)$ & & \\
\hline \multicolumn{5}{|l|}{6 hour } \\
\hline Mean \pm SD & $67.46 \pm 24.85$ & $172.22 \pm 120.50$ & \multirow{2}{*}{-2.984} & \multirow{2}{*}{$0.003(\mathrm{HS})$} \\
\hline Median (range) & $66(35-124)$ & $132(30-480)$ & & \\
\hline \multicolumn{5}{|l|}{24 hour } \\
\hline Mean \pm SD & $83.07 \pm 32.17$ & $444.50 \pm 386.28$ & \multirow{2}{*}{-3.344} & \multirow{2}{*}{$0.001(\mathrm{HS})$} \\
\hline Median (range) & $83(35-150)$ & $310(35-1404)$ & & \\
\hline \multicolumn{5}{|l|}{$3^{\text {rd day }}$} \\
\hline Mean \pm SD & $123.76 \pm 83.80$ & $680.50 \pm 953.83$ & \multirow{2}{*}{-3.403} & \multirow{2}{*}{$0.001(\mathrm{HS})$} \\
\hline Median (range) & $105(33-310)$ & $502.50(40-4270)$ & & \\
\hline \multicolumn{5}{|l|}{$5^{\text {th }}$ day } \\
\hline Mean \pm SD & $104.23 \pm 65.35$ & $366.66 \pm 305.35$ & \multirow{2}{*}{-3.364} & \multirow{2}{*}{$0.001(\mathrm{HS})$} \\
\hline Median (range) & $77(32-250)$ & $281.50(38-1200)$ & & \\
\hline Test $\ddagger$ & 18.892 & 51.244 & & \\
\hline P - value (Sig.) & $0.001(\mathrm{HS})$ & $<0.001$ (HS) & & \\
\hline
\end{tabular}

$\mathrm{p}<0.05$ is significant; Sig.: significance.

Table 12: Relation between severity liver injury and AST.

\begin{tabular}{|c|c|c|c|c|}
\hline AST & $\begin{array}{l}\text { Minor liver injury } \\
(\mathrm{N}=13)\end{array}$ & $\begin{array}{l}\text { Major liver injury } \\
(\mathrm{N}=18)\end{array}$ & Test• & p-value (Sig.) \\
\hline \multicolumn{5}{|l|}{ Initial } \\
\hline Mean \pm SD & $57.46 \pm 25.77$ & $107.55 \pm 47.17$ & \multirow{2}{*}{-2.525} & \multirow{2}{*}{$0.012(\mathrm{~S})$} \\
\hline Median (Range) & $46(33-110)$ & $102.50(22-199)$ & & \\
\hline \multicolumn{5}{|l|}{6 hour } \\
\hline Mean \pm SD & $79.76 \pm 37.16$ & $195.05 \pm 128.14$ & \multirow{2}{*}{-3.104} & \multirow{2}{*}{0.002 (HS) } \\
\hline Median (Range) & $70(40-160)$ & $176(27-564)$ & & \\
\hline \multicolumn{5}{|l|}{24 hour } \\
\hline Mean \pm SD & $114.76 \pm 68.17$ & $445.66 \pm 314.29$ & \multirow{2}{*}{-3.464} & \multirow{2}{*}{0.001 (HS) } \\
\hline Median (Range) & $85(50-299)$ & $360(35-1140)$ & & \\
\hline \multicolumn{5}{|l|}{$3^{\text {rd day }}$} \\
\hline Mean \pm SD & $177.84 \pm 141.93$ & $653.11 \pm 688.05$ & \multirow[b]{2}{*}{-3.123} & \multirow{2}{*}{$0.002(\mathrm{HS})$} \\
\hline Median (Range) & $105(55-560)$ & $535(39-3157)$ & & \\
\hline \multicolumn{5}{|l|}{$5^{\text {th }}$ day } \\
\hline Mean \pm SD & $144.38 \pm 113.89$ & $390.83 \pm 308.95$ & \multirow{2}{*}{-2.462} & \multirow[b]{2}{*}{$0.014(\mathrm{~S})$} \\
\hline Median (Range) & $100(42-450)$ & $337.50(44-1163)$ & & \\
\hline Test $\downarrow$ & 35.815 & 48.578 & & \\
\hline p-value (Sig.) & $<0.001$ (HS) & $<0.001$ (HS) & & \\
\hline
\end{tabular}

$\mathrm{p}<0.05$ is significant; Sig.: significance.

Table 13: Relation between severity of liver injury and laboratory investigation.

\begin{tabular}{|c|c|c|c|c|c|c|}
\hline \multirow{3}{*}{ Liver function tests } & \multirow{2}{*}{\multicolumn{2}{|c|}{$\begin{array}{l}\text { Minor liver injury } \\
(\mathbf{N}=\mathbf{1 3})\end{array}$}} & \multirow{2}{*}{\multicolumn{2}{|c|}{$\begin{array}{l}\text { Major liver injury } \\
(\mathbf{N}=\mathbf{1 8})\end{array}$}} & \multirow{3}{*}{ Test } & \multirow{3}{*}{ p-value (Sig.) } \\
\hline & & & & & & \\
\hline & No. & $\%$ & No. & $\%$ & & \\
\hline \multicolumn{7}{|l|}{ ALT } \\
\hline Normal & 2 & $15.4 \%$ & 1 & $5.6 \%$ & \multirow{2}{*}{0.834} & \multirow{2}{*}{$0.361(\mathrm{NS})$} \\
\hline Abnormal & 11 & $84.6 \%$ & 17 & $94.4 \%$ & & \\
\hline \multicolumn{7}{|l|}{ AST } \\
\hline Normal & 0 & $0 \%$ & 1 & $5.6 \%$ & \multirow{2}{*}{0.746} & \multirow{2}{*}{$0.388(\mathrm{NS})$} \\
\hline Abnormal & 13 & $100 \%$ & 17 & $94.4 \%$ & & \\
\hline \multicolumn{7}{|l|}{ Bilirubin } \\
\hline Normal & 12 & $92.3 \%$ & 11 & $61.1 \%$ & 3.837 & $0.050(\mathrm{NS})$ \\
\hline
\end{tabular}




\begin{tabular}{|c|c|c|c|c|c|c|}
\hline Abnormal & 1 & $7.7 \%$ & 7 & $38.9 \%$ & & \\
\hline \multicolumn{7}{|l|}{ ALP } \\
\hline Normal & 11 & $84.6 \%$ & 7 & $38.9 \%$ & \multirow{2}{*}{6.482} & \multirow{2}{*}{$0.011(\mathrm{~S})$} \\
\hline Abnormal & 2 & $15.4 \%$ & 11 & $61.1 \%$ & & \\
\hline \multicolumn{7}{|l|}{ LDH } \\
\hline Normal & 4 & $30.8 \%$ & 2 & $11.1 \%$ & \multirow{2}{*}{1.869} & \multirow[b]{2}{*}{$0.172(\mathrm{NS})$} \\
\hline Abnormal & 9 & $69.2 \%$ & 16 & $88.9 \%$ & & \\
\hline \multicolumn{7}{|l|}{ Albumin } \\
\hline Normal & 13 & $100 \%$ & 12 & $66.7 \%$ & \multirow{2}{*}{5.373} & \multirow{2}{*}{$0.020(\mathrm{~S})$} \\
\hline Abnormal & $0 \%$ & $0 \%$ & 6 & $33.3 \%$ & & \\
\hline \multicolumn{7}{|c|}{ Haemoglobin } \\
\hline Normal & 8 & $61.5 \%$ & 8 & $44.4 \%$ & \multirow{2}{*}{3.363} & \multirow{2}{*}{$0.122(\mathrm{~S})$} \\
\hline Abnormal & 5 & $38.5 \%$ & 10 & $55.6 \%$ & & \\
\hline \multicolumn{7}{|c|}{ Haematocrit } \\
\hline Normal & 9 & $69.2 \%$ & 9 & $50 \%$ & \multirow{2}{*}{2.870} & \multirow{2}{*}{$0.112(\mathrm{NS})$} \\
\hline Abnormal & 4 & $30.2 \%$ & 9 & $50 \%$ & & \\
\hline
\end{tabular}

\section{DISCUSSION}

Our study was a prospective study, showing the diagnostic value of liver enzymes (which are alanine aminotransferase, aspartate amino-transferase, lactate dehydrogenase and alkaline phosphatase, albumen) in diagnosis and assessment of severity of liver injuries which were graded and classified into minor and major by American association for the surgery of trauma (AAST) organ injury scale. ${ }^{8}$

The study was carried out on 62 patients, the median age was 29 years (ranges from 3 to 65 years), and the peak incidence $(27.4 \%)$ was in the third decades of life (20-30) years, the less incidence was in seventh decades of life $(3 \%)$ as old age persons have less activity. Males were more affected than females (male represented 73\%).

In our study, road traffic accident was the commonest mechanism of injury in most of cases, RTA represented in thirty eight patients $(61.3 \%)$.

In our study, the most grades of hepatic injuries were grade III. Nine patients were grade III (29\%), then grade II in eight patients $(25.8 \%)$, then grade IV in six patients $(19.3 \%)$, grade I in five patients $(16.2 \%)$ and grade $\mathrm{V}$ in three patients $(9.6 \%)$, lastly no patient for grade VI as it incompatible with life. This is almost in agreement with Fabian TC al who studied in a prospective study factors affecting morbidity following hepatic trauma in 482 patients. ${ }^{9}$ Liver trauma is graded from I to VI. 51 patients $(11 \%)$ were graded I , 171 patients $(35 \%)$ were grade II , 180 patients $(37 \%)$ were graded III 42 patients $(9 \%)$ were grade IV, 28 patients $(6 \%)$ were grade $\mathrm{V}$, six patients (1\%) were VI .

As regard abdominal examination; in our study, the main clinical finding in abdominal palpation were tenderness and rigidity in both group then abdominal wall contusion in inspection This is also coincides with Schurink GW et al who reported that physical examination of the abdomen was beneficial in blunt abdominal trauma, 20 patients out of 23 patients $(87 \%)$ of patients with major blunt abdominal injuries had rigidity and guarding in abdominal examination. ${ }^{10}$

Our results showed that the most 2 dependable liver enzymes were alanine aminotransferase (ALT) and, aspartate aminotransferase (AST). As regard alanine aminotransferases (ALT), Our study showed that serum ALT was raised in about twenty eight cases from thirty one cases of liver injury (sensitivity $=90.3 \%$, Table 8), Only four patients with raised serum ALT did not have liver trauma (specificity $=87 \%$, Table 8), positive predictive values $87.5 \%$, negative predictive value $90 \%$ (Table 8), it was showed initial median value after injury $66 \mathrm{IU} / \mathrm{L}$ in liver group and increase gradually to reach maximum level mainly within $3^{\text {rd }}$ day of injury with median value about 190 IU/L (Table 5), it also showed that in minor hepatic injuries initial median value less than $87 \mathrm{IU} / \mathrm{L}$ and level more than $87 \mathrm{IU} / 1$ mainly associated with major hepatic injury (Table 11).

As regard asparate aminotransferases (AST), our study showed serum AST was raised in about twenty nine cases from thirty one cases of liver injury (sensitivity $=93.5 \%$, Table 8), only seven patients with raised serum AST did not have liver trauma (specificity $=77.4 \%$, Table 8 ), positive predictive values $81.1 \%$, negative predictive value $93 \%$ (Table 8 ), it was showed initial median value immediately after injury $81 \mathrm{IU} / \mathrm{L}$ in liver group and increase gradually to reach maximum level mainly within 3rd day of injury with median value about 290 IU/L (Table 6), it also showed that in minor hepatic injuries initial median value less than 102 IU/L, and level more than 102 IU/l mainly associated with major hepatic injury (Table 12).

According to Bilgic I et al, he found, abnormal hepatic transaminases and LDH levels are associated with liver injury. ${ }^{11}$ ALT $\leq 76 \mathrm{U} / \mathrm{L}, \mathrm{AST}<130 \mathrm{U} / \mathrm{L}$, and $\mathrm{LDH} \leq 410$ $\mathrm{UL} / \mathrm{L}$ are predictive of low grade liver injury, While 
serum liver levels above these were strongly associated with major hepatic injury.

According to TIAN Z, et al, who studied role of elevated liver transaminase levels in the diagnosis of liver injury after blunt abdominal trauma, he suggested that in patients with blunt abdominal trauma, abnormal transaminase levels are associated with liver injury. ${ }^{12}$ Patients with ALT > $57 \mathrm{U} / \mathrm{l}$ and AST >113 U/l are strongly associated with liver injury.

And according to Bevan $\mathrm{C}$ et al who studied the possibility of ALT to predict patients with liver injury. ${ }^{13}$ He found that in 51 patients with liver injuries and 65 patients with other intra-abdominal injuries, when ALT level >104 IU/L have a sensitivity of $96 \%$ and a specificity of $80 \%$ of having liver injuries.

Tan KK et al who studied the role of hepatic enzymes in the diagnosis of hepatic injury, he found raised ALT was strongly associated with presence of hepatic injuries. ${ }^{14}$ This relation was also seen in patients with raised AST > 2 times. This difference was not seen in both bilirubin and ALP. Also, ALT > 2 times was associated with major hepatic injuries, while patients with simultaneous raised AST > 2 times and ALT > 2 times had a stronger association for major hepatic injuries.

In our study, It showed that LDH is one of liver enzymes can be used to predict the presence of liver injuries by high sensitivity of $80.6 \%$ but low specificity of $29 \%$ (Table 19) and values more than 503 IU/L were associated with major hepatic injuries . This is agreement with Tan KK et al, who documented that sensitivity of lactate dehydrogenase in diagnosis of liver trauma is $95.7 \%$ with specificity $25 \%$, The presence of LDH in many body tissues other than the liver makes the low specificity of LDH in detection of liver injury. ${ }^{14}$

In our study serum bilrubin, alkaline phosphatase enzymes and serum albumen were usually normal in both liver injury and non-liver injury group and there were not related to any liver trauma, with low sensitivity and specificity in diagnosis of liver trauma. This is agreement with Tan KK et al, who studied the role of hepatic enzymes in the diagnosis of hepatic injury, who documented that both ALP and bilirubin were not useful in the screening of hepatic injuries. ${ }^{14}$

In our study twenty four patients out of thirty one patients with liver injury $(77.4 \%)$ had done C.T abdomen , and only seven patients $(22.6 \%)$ didn't do CT due hemodynamic instability or penetrating injury which need urgent exploration. As regard that, twenty four patients with liver injuries were diagnosed and graded by C.T, while in the remaining liver injuries, their grading and diagnosis were done intra-operative.

According to Tan KK et al who studied role of hepatic enzymes in the diagnosis of hepatic injury, he found that cases of liver laceration C.T was performed in $89.1 \%$, while $10.9 \%$ not performed C.T due to hemodynamically instability. ${ }^{14}$

As regard of management of liver trauma, in our study out of 31 patients with liver injuries; twenty two patients (71\%) underwent successful conservative management, while nine patients $(29 \%)$ underwent surgical management.

According to Srivastava AR et al; serum ALT- A marker of liver injury and a guide to assessment of its severity, ten patients $(32 \%)$ out of thirty one patients with bunt liver injury managed with surgical intervention and twenty one patients $(68 \%)$ conservative management. ${ }^{15}$

In our study the main organ had been injured in non-liver injury group is spleen followed by renal organ and this was accepted with Meheta $\mathrm{N}$ et al, who mentioned in his A retrospective study of 71 cases of blunt abdominal trauma patients that spleen is the main organ injured about $53 \%$ followed by liver $35 \%{ }^{16}$

In our study patients with liver injury tended to be significantly more severely injured than those without liver injury, median ISS, LOS in ICU were more in liver injury group. There were three deaths included in our study two of them were related to liver injury group and the last in non-liver injury group.

\section{Recommendation}

We advocate the use of serum ALT and AST as part of the initial assessment of patients after abdominal trauma in centers with limited resources.

All patients with raised patients with ALT more than two times normal level and AST more than two and half times normal level must be excluded from possessing severe hepatic injuries and should be managed accordingly to that in trauma centers with available resources.

Our series also suggested that patients with normal ALT, AST were unlikely to possess significant hepatic injuries and would not require further screening for hepatic injuries. Even if hepatic injuries were present, it is more likely to be minor and can be managed conservatively. It found that using liver enzymes to predict the need for CT scanning could help us in decreasing time, cost and achieving safety in the work-up of stable patients with potential liver injury.

\section{CONCLUSION}

An abnormal ALT and AST can imply the presence of hepatic injuries in abdominal trauma patients, while the level of the abnormality can determine the severity of the insult to the liver. 
We can conclude that ALT is more specific for liver injury more than AST, Although AST show high sensitivity for liver injury than ALT.

\section{Funding: No funding sources}

Conflict of interest: None declared

Ethical approval: The study was approved by the institutional ethics committee

\section{REFERENCES}

1. Hsu JM, Joseph AP, Tarlinton LJ, Macken L, Blome $S$. The accuracy of focused assessment with sonography in trauma (FAST) in blunt trauma patients. Experience of an Australian major trauma service Injury. 2006;38:71-5.

2. Centers for disease control and prevention. National center for injury prevention and control. Web-based injury statistics query and reporting system, 2007. Available at http://www.cdc.gov /injuty /wisqars.

3. Piper GL, Peitzman AB. Current management of hepatic trauma. Surg Clin North Am. 2010;90(4):775-85.

4. Corthren CC, Moore EE. Hepatic trauma. European journal of trauma and emergency surgery. 2008;34(4):336-9.

5. Ritchie AH, Williscroft DM. Elevated liver enzymes as a predictor of liver injury in stable blunt abdominal trauma patients: case report and systematic review of the literature. Can J Rural Med. 2006;11:283-7.

6. Elbaih A, Ali M, Attia Y. Accuracy of Predictive Factors and Focused Assessment with Sonography for Trauma (FAST) in Management of Adult Blunt Abdominal Trauma and Its Outcome. IJSM. 2016;2(2):58-63.

7. Rosalki SB, Mcintyre N. Biochemical investigations in the management of liver disease. Oxford textbook of clinical hepatology, $2^{\text {nd }}$ ed. New York; Oxford university press; 1999:503-521.

8. Moore EE, Cogbill TH, Jurkovich GJ. Organ injury scaling: spleen and liver (1994 revision). J Trauma. 1995;38(3):323-4.

9. Fabian TC, Croce MA, Standford GG, Payne LW, Mangiante CE, Voeller GR, et al. Factor affecting morbidity following hepatic trauma. Ann Surg. 1991;213:540-8.

10. Schurink GW, Bode PJ, van Luijt PA, van Vugt AB. The value of physical examination in the diagnosis of patients with blunt abdominal trauma. Pub med. 1997;28(4):261-5.

11. Bilgic IC, Gelecek S. Predictive value of liver transaminases level in abdominal trauma. Am J Emerg Med. 2014;32(issue 7):705-8.

12. Tian Z, Liu H, Su X, Fang Z, Dong Z, Yu C, et al. Liver transaminases in diagnosis of liver injury, Experimental and therapeutic medicine. 2012;4:25560.

13. Bevan CA, Palmer CS, Sutcliffe JR, Rao P, Gibikote S, Crameri J. Blunt abdominal trauma in children: how predictive is ALT for liver injury. Emergency Medicine Journal. 2009;26(4):283-8.

14. Tan KK, Bang SL, Vijayan A, Chiu MT. Hepatic enzymes have a role in the diagnosis of hepatic injury after blunt abdominal trauma. Injury Int $\mathbf{J}$. 2009;40:978-83.

15. Srivastava AR, Kumar S, Agarwal GG, Ranjan P. Blunt abdominal injury: ALT-A marker of liver injury and a guide to assessment of its severity. Injury. 2007;38:1069-74.

16. Mehta N, Babu S, Venugopal K. An experience with blunt abdominal trauma: evaluation, management and outcome. Clin pract. 2014;4(2):599.

Cite this article as: Elbaih AH, Ali MA, Abozaid A, Saady BM, Farrag SH. Role of elevated liver transaminase enzymes in diagnosis of liver injury in cases after abdominal trauma. Int Surg J 2016;3:1184-92. 